\title{
Incidence and risk of thromboembolism associated with bevacizumab in patients with non-small cell lung carcinoma
}

\author{
Li-Juan Li" ${ }^{1 \#}$, Di-Fei Chen ${ }^{1 \#}$, Guo-Feng Wu ${ }^{2 \#}$, Wei-Jie Guan ${ }^{3}$, Zheng Zhu ${ }^{3}$, Yi-Qian Liu ${ }^{3}$, Guo-Ying Gao ${ }^{3}$, \\ Yin-Yin Qin ${ }^{3}$, Nan-Shan Zhong ${ }^{3}$
}

${ }^{1}$ Nanshan School, Guangzhou Medical University, Guangzhou 511436, China; ${ }^{2}$ The 3rd Affiliated Hospital of Guangzhou Medical University LiWan Hospital, Guangzhou 510170, China; ${ }^{3}$ State Key Laboratory of Respiratory Disease, The First Affiliated Hospital of Guangzhou Medical University, National Clinical Research Center for Respiratory, Guangzhou Institute of Respiratory Health, Guangzhou 510120, China

Contributions: (I) Conception and design: LJ Li, DF Chen, GF Wu, YY Qin, NS Zhong; (II) Administrative support: LJ Li, DF Chen, GF Wu, YY Qin; (III) Provision of study materials or patients: None; (IV) Collection and assembly of data: DF Chen, LJ Li, GF Wu, Z Zhu, YQ Liu, GY Gao; (V) Data analysis and interpretation: DF Chen, LJ Li, GF Wu, WJ Guan, YY Qin; (VI) Manuscript writing: All authors; (VII) Final approval of manuscript: All authors.

\# These authors contributed equally to this work.

Correspondence to: Yin-Yin Qin, PhD. State Key Laboratory of Respiratory Disease, The First Affiliated Hospital of Guangzhou Medical University, National Clinical Research Center for Respiratory, Guangzhou Institute of Respiratory Health, 151 Yanjiang Road, Guangzhou 510120, China. Email: 13610047638@163.com; Nan-Shan Zhong, MD. State Key Laboratory of Respiratory Disease, The First Affiliated Hospital of Guangzhou Medical University, National Clinical Research Center for Respiratory, Guangzhou Institute of Respiratory Health, 151 Yanjiang Road, Guangzhou 510120, China. Email: nanshan@vip.163.com.

Background: Bevacizumab, a recombinant humanized monoclonal antibody against vascular endothelial growth factor (VEGF), is effective for the treatment of advanced non-small cell lung cancer (NSCLC). However, severe adverse events (AEs) have been reported in NSCLC patients treated with bevacizumab. Currently, the contribution of Bevacizumab to thromboembolism is still controversial. We conducted a study to determine the overall risk and incidence of thromboembolism with bevacizumab in NSCLC patients.

Methods: Electronic databases such as the PubMed, Web of Science and Cochrane Library were searched for related trials. Statistical analyses were conducted to calculate the overall incidence rates, odds ratios (ORs), and $95 \%$ confidence intervals (CIs) by using either random-effect or fixed-effect models depending on the heterogeneity. We also used trial sequence analysis (TSA) to verify the pooled result.

Results: A total of 3,555 subjects from nine studies were included. The overall incidence of thromboembolism events in NSCLC patients treated with bevacizumab was 4.8\% (95\% CI: 1.9-7.7\%). Without bevacizumab, this incidence was 2.9\% (95\% CI: 0.6-5.1\%). Bevacizumab use was associated with a significantly increased risk in thromboembolism events ( $\mathrm{OR}=1.74 ; 95 \% \mathrm{CI}: 1.15-2.62 ; \mathrm{P}=0.008$ ). Subgroup analysis based on the doses showed that bevacizumab administered at $15 \mathrm{mg} / \mathrm{kg}(\mathrm{OR}=1.81 ; 95 \%$ CI: 1.14-2.86; $\mathrm{P}=0.012$ ), but not $7.5 \mathrm{mg} / \mathrm{kg}$ (OR =1.32; 95\% CI: 0.78-2.24; $\mathrm{P}=0.296$ ), increased the risk of thromboembolism.

Conclusions: Bevacizumab is associated with a significantly increased risk of thromboembolism development in NSCLC patients. It may have dose-toxicity relationship and low dose of bevacizumab may be a better choice for NSCLC patients, with equal efficacy and low hazard of thromboembolism events.

Keywords: Bevacizumab; thromboembolism; lung cancer; meta-analysis

Submitted Jan 18, 2018. Accepted for publication Jul 02, 2018.

doi: $10.21037 /$ jtd.2018.07.09

View this article at: http://dx.doi.org/10.21037/jtd.2018.07.09

(c) Journal of Thoracic Disease. All rights reserved.

jtd.amegroups.com

J Thorac Dis 2018;10(8):5010-5022 


\section{Introduction}

Lung cancer is the leading cause of cancer-related death worldwide (1). Patients with non-small cell lung cancer (NSCLC), which accounts for $85 \%$ of all lung cancer cases, often present with advanced disease at initial diagnosis (2). Platinum-based doublet chemotherapy has constituted the first-line treatment for advanced NSCLC patients with good performance status, however, only less than $40 \%$ of patients showed significant tumor size reduction $(3,4)$. The standard cytotoxic chemotherapy confers limited efficacy, so there is a need for exploring novel therapeutic approaches. Angiogenesis, a prerequisite for proliferation and metastasis of cancer cells, results from the interactions between the tumor and its growing environment including the vascular endothelial growth factor (VEGF) (5). Through binding with VEGF ligand or receptor (VEGFR), the anti-VEGF antibody can inhibit the tumor-induced angiogenesis and tumor growth, without compromising the function of normal tissue vasculature. Both VEGF ligand and receptor are promising targets for anticancer therapy with anti-angiogenic activity (6). Bevacizumab, a recombinant humanized monoclonal antibody that binds to VEGF, is the first effective angiogenesis inhibitor for solid tumors (7). Several clinical trials have proved the clinical benefits in patients with advanced NSCLC (5). The phase III randomized trial (ECOG4599) showed that addition of bevacizumab to a standard platinum-based chemotherapy regimen improved overall survival (OS) and prolonged progression-free survival (PFS) in patients with advanced non-squamous NSCLC (8).

Overall, bevacizumab has been well tolerated, with most adverse events (AEs) being rated as mild to moderate and are manageable with proper interventions $(2,9)$. However, severe toxicities (including hypertension, proteinuria, bleeding events, and neutropenia) have been reported in NSCLC patients treated with bevacizumab in combination with chemotherapy, (8,10-12). Thromboembolism is also related to the extensive use of bevacizumab. In a metaanalysis, thromboembolism was associated with increased incidence of thrombosis, and compared with non-Asian patients, Asian populations had a higher incidence of developing mild or moderate thromboembolism rather than severe thromboembolism (13). However, in two other meta-analyses, no significant differences were found for thromboembolic events with the addition of bevacizumab therapy in patients with advanced NSCLC (14,15). The specific role of bevacizumab in the development of severe
AEs in patients with NSCLC remains controversial. Moreover, severe thromboembolism events, (such as pulmonary embolism) can be life-threatening. Therefore, clinicians should be fully aware of screening for severe AEs associated with bevacizumab therapy. An analysis on the risk of thromboembolism in patients receiving bevacizumab treatment may offer additional insights into the relevant risk factors, proper monitoring, implementation of management strategies, and selection of the standard anti-cancer medications. Therefore, we conducted a meta-analysis of randomized controlled trials (RCTs) to investigate the risk of thromboembolism with add-on bevacizumab therapy among patients with advanced NSCLC.

\section{Methods}

\section{Search strategy and study selection}

We searched articles from electronic databases including Cochrane Central Register of Controlled Trials (CENTRAL), PubMed (up to June 2017), Web of Science (up to June 2017), and Google Scholar with search terms "bevacizumab", "avastin", "lung cancer", "lung carcinoma", "NSCLC", and "randomized controlled trial". Additionally, we searched for relevant abstracts and virtual meeting presentations from the American Society of Clinical Oncology (http://www.asco.org/ASCO) published up to 2017. If studies had partly overlapped patients, only the most original or complete study was selected. For clinical trials with missing data, we searched the clinical trialregistration website (ClinicalTrials.gov) to obtain relative information.

Clinical trials that satisfy the following criteria were eligible for the present meta-analysis: (I) prospective phase II or III randomized controlled trials (RCTs); (II) patients with advanced NSCLC; (III) patients without a history of cardiovascular disease, anticoagulation therapy and antiplatelet therapy; (IV) participants assigned to treatment with or without bevacizumab $(7.5 \mathrm{mg} / \mathrm{kg}$ and/or $15 \mathrm{mg} / \mathrm{kg})$ in addition to concurrent therapy using a chemotherapeutic or biological agent; and (V) available data regarding adverse effects with concrete sample size of thromboembolism events including arterial or venous thromboembolic events, and pulmonary embolism.

\section{Data extraction}

All data were independently extracted by two investigators 


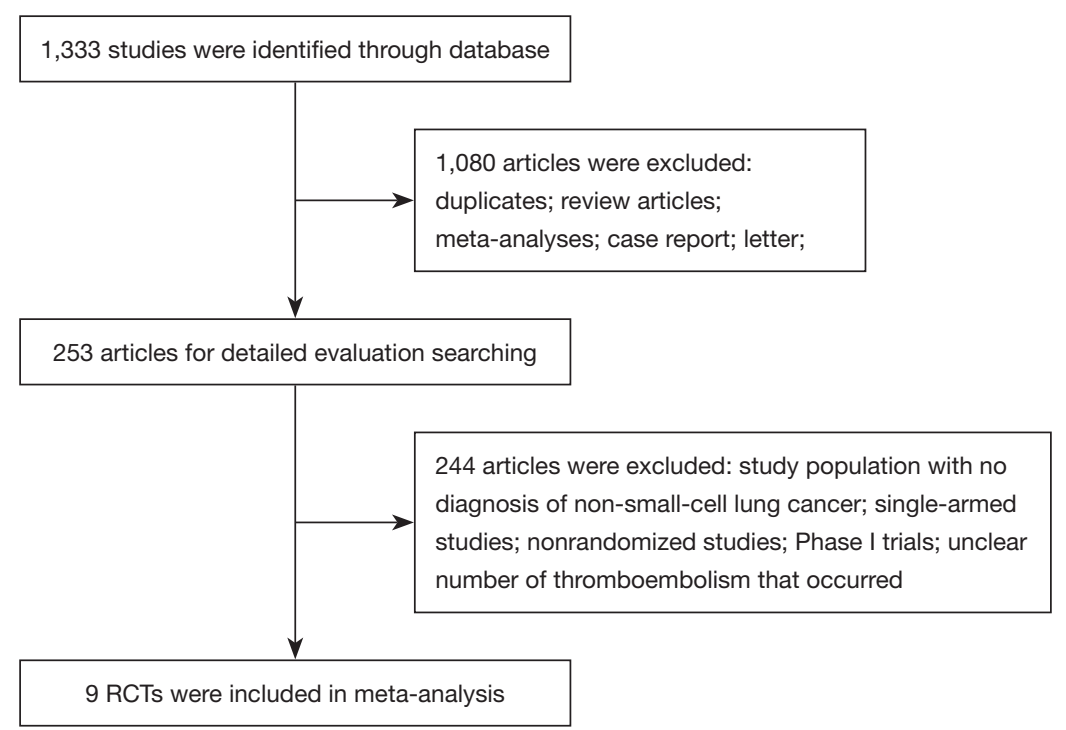

Figure 1 Flowchart demonstrating the study-selection process. RCTs, randomized controlled trials.

(LJ Li and DF Chen). Disagreements were resolved by discussion between the two reviewers and in case no consensus was made an independent expert was further consulted. For each study, the following information was extracted: first author, year of publication, number of the patients in treatment and control groups, treatment arms, the number of thrombosis events in both groups and the dosage of bevacizumab.

\section{Statistical analysis}

To calculate the odds ratio (OR), we only compared the patients who received bevacizumab with those in the control arm within the same trial. For four studies which reported no events in treatment or control arm, we used the classic half-integer correction to calculate OR and variance (16). The Cochran's $\chi^{2}$ test and I $I^{2}$ was used to examine the heterogeneity among the effect estimates, with $\mathrm{P}<0.05$ or $\mathrm{I}^{2}>50 \%$ was considered statistically significant for the heterogeneity. Fixed-effects model was used in case of no significant heterogeneity. $\mathrm{P}<0.05$ denoted statistical significance for two-sided tests. Potential publication bias was evaluated for thrombotic events by using Egger's test. All statistical analyses were conducted with STATA (Version 12.0). Trial sequence analysis (TSA) was conducted to primary outcome by TSA software, version 0.9 .5 .5 beta; Copenhagen Trial Unit.

\section{Risk of bias assessment}

Two authors (LJ Li and DF Chen) independently evaluated the risk of bias of included RCTs according to the recommendations of the Cochrane Handbook for Systematic Reviews of Interventions (17). The assessment criteria included the following seven domains: random sequence generation, allocation sequence concealment, blinding of participants and personnel, blinding of outcome assessment, incomplete outcome data, selective outcome reporting, and other biases. All domains were evaluated as "low," "high," or "unclear" according to the Cochrane Handbook for Systematic Reviews of Interventions (version 5.3.0) (17) and the risks of bias were drawn by the Review Manager 5.3.0 software (The Nordic Cochrane Centre, The Cochrane Collaboration, Copenhagen, Denmark).

\section{Results}

\section{Study characteristics}

Nine case-control studies $(8,11,18-24)$ including 3,555 patients (1,983 cases and 1,572 controls) met the inclusion criteria. The process of data extraction was shown in Figure 1. Table 1 demonstrates the studies identified and the baseline characteristics. The sample sizes of the nine studies ranged from 72 to 867 . All studies focused on patients with 


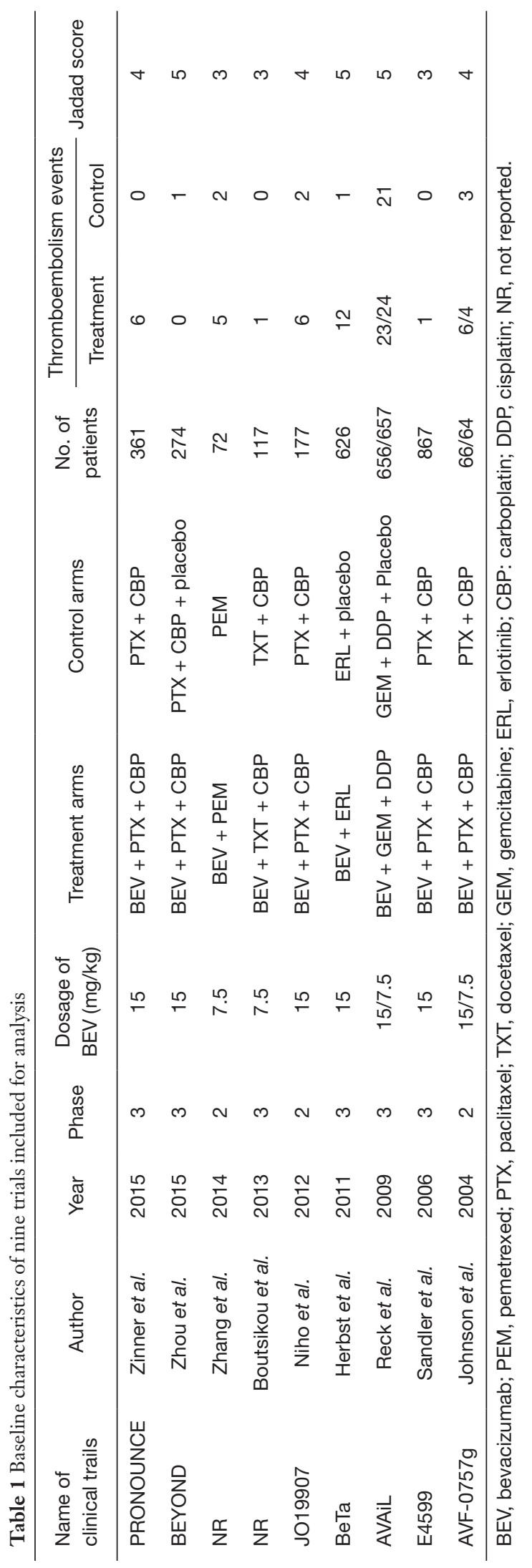

NSCLC, under critical histologic diagnosis. Bevacizumab had two main doses, low $(7.5 \mathrm{mg} / \mathrm{kg}$ for three weeks) and high dose ( $15 \mathrm{mg} / \mathrm{kg}$ for three weeks).

\section{Risk of bias}

Figure 2 shows the details of the risk of bias assessment for all included studies. Randomized sequence generation was implemented adequately in 4 studies $(11,19,20,24)$; the other 5 studies $(8,18,21-23)$ had an unclear risk of bias. In contrast to other meta-analysis of RCT, we studied the thromboembolism event of bevacizumab, which was not likely to be influenced by the lack of blinding. Therefore, despite that we have included some open-label trials, the risk of performance bias was still rated as "low". The overall kappa value regarding the evaluation of risk of bias of included RCTs was $>0.95$, indicating an excellent degree of agreement between the 2 raters.

\section{Incidence of thromboembolism}

We calculated the incidence of thromboembolism. In treatment group, the incidence ranged from $0 \%$ to $15.15 \%$. Based on the data from trials included (with the incidence of thromboembolism of $0 \%$ had been excluded), the calculated overall incidence of thromboembolism was $4.8 \%$ [95\% confidence intervals (CIs): 1.9-7.7\%] according to random effects model $\left(\mathrm{I}^{2}=91.0, \mathrm{P}<0.001\right)$. In control group, the incidence of thromboembolism ranged from $0 \%$ to $9.6 \%$. The overall incidence in control group was 2.9\% (95\% CI: 0.6-5.1\%) according to random-effects model. We did the same calculation in different dosage groups of bevacizumab. At high dosage, the overall incidences of thromboembolism in two the arms were $3.9 \%$ (95\% CI: $1.0-6.7 \%$ ) in treatment group) and 2.7\% (95\% CI: $0.3-5.0 \%$ ) in control group. At low dosage, the overall incidences were $6.8 \%$ (95\% CI: $2.0-11.6 \%)$ in treatment group and 6.5\% (95\% CI: $4.1-8.9 \%)$ in control group.

\section{Relative risk of thromboembolism}

The events of thromboembolism in each study are described as the number of cases in Table 1. We determined the risk of thromboembolism according to the OR. The results showed no significant heterogeneity among studies, so we used fixed-effect model $\left(\mathrm{I}^{2}=9.4 \%, \mathrm{P}=0.356\right)$. Metaanalysis showed that the combination of bevacizumab with chemotherapy was associated with higher risks of 
A

Random sequence generation (selection bias) Allocation concealment (selection bias) Blinding of participants and personnel (performance bias) Blinding of outcome assessment (detection bias) Incomplete outcome data (attrition bias) Selective reporting (reporting bias) Other bias

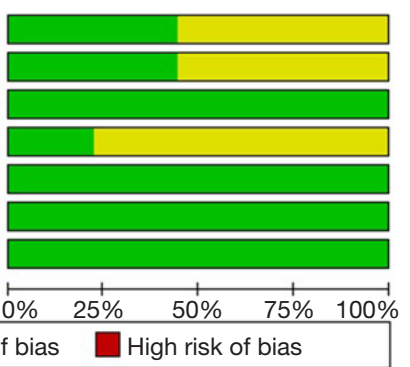

B

$\square$ Low risk of bias $\quad \square$ Unclear risk of bias $\quad \square$ High risk of bias

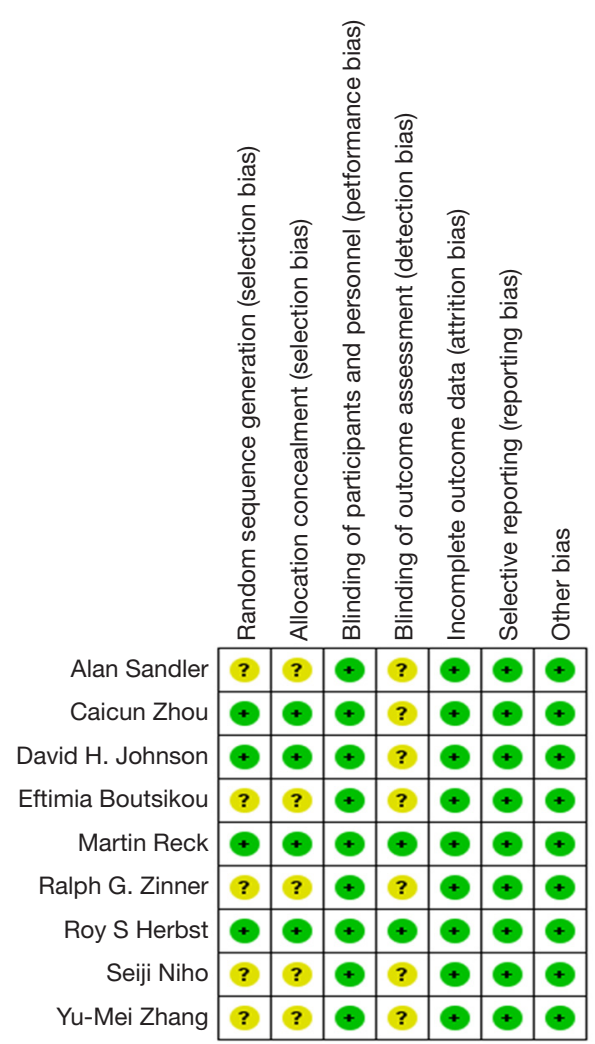

Figure 2 Summary of risk of bias assessment. (A) The risk of bias graph; (B) risk of bias of included in randomized controlled trials and summary of the included studies. +, no bias; -, bias; ?, bias unknown.

thromboembolism events (OR $=1.74 ; 95 \%$ CI: 1.15-2.62; $\mathrm{P}=0.008$ ) (Figure 3).

\section{Subgroup analysis of doses}

We studied different doses of bevacizumab $(7.5 \mathrm{mg} / \mathrm{kg}$ and $15 \mathrm{mg} / \mathrm{kg}$ ) to further elucidate the effects in subgroups. There were seven trials which studied high doses of bevacizumab, with the meta-analysis demonstrating that $15 \mathrm{mg} / \mathrm{kg}$ bevacizumab may increase the risk of thromboembolism (OR $=1.81 ; 95 \% \mathrm{CI}$ : $1.14-2.86 ; \mathrm{P}=0.012)$ (Figure 4). Only four trials including 846 patients studied low doses of bevacizumab. Meta-analysis showed that there were no significant differences between cases and controls in thromboembolism hazard ( $\mathrm{OR}=1.32$; 95\% CI: 0.78-2.24; $\mathrm{P}=0.296$ ) (Figure 5).

\section{Sensitive analysis}

Considering that a RCTs with a significant weight (19) were identified in the meta-analysis, a sensitivity analysis was conducted to assess the stability of the results of this metaanalysis by excluding the study with the greatest weight study. The sensitivity analysis indicated that no exclusion 


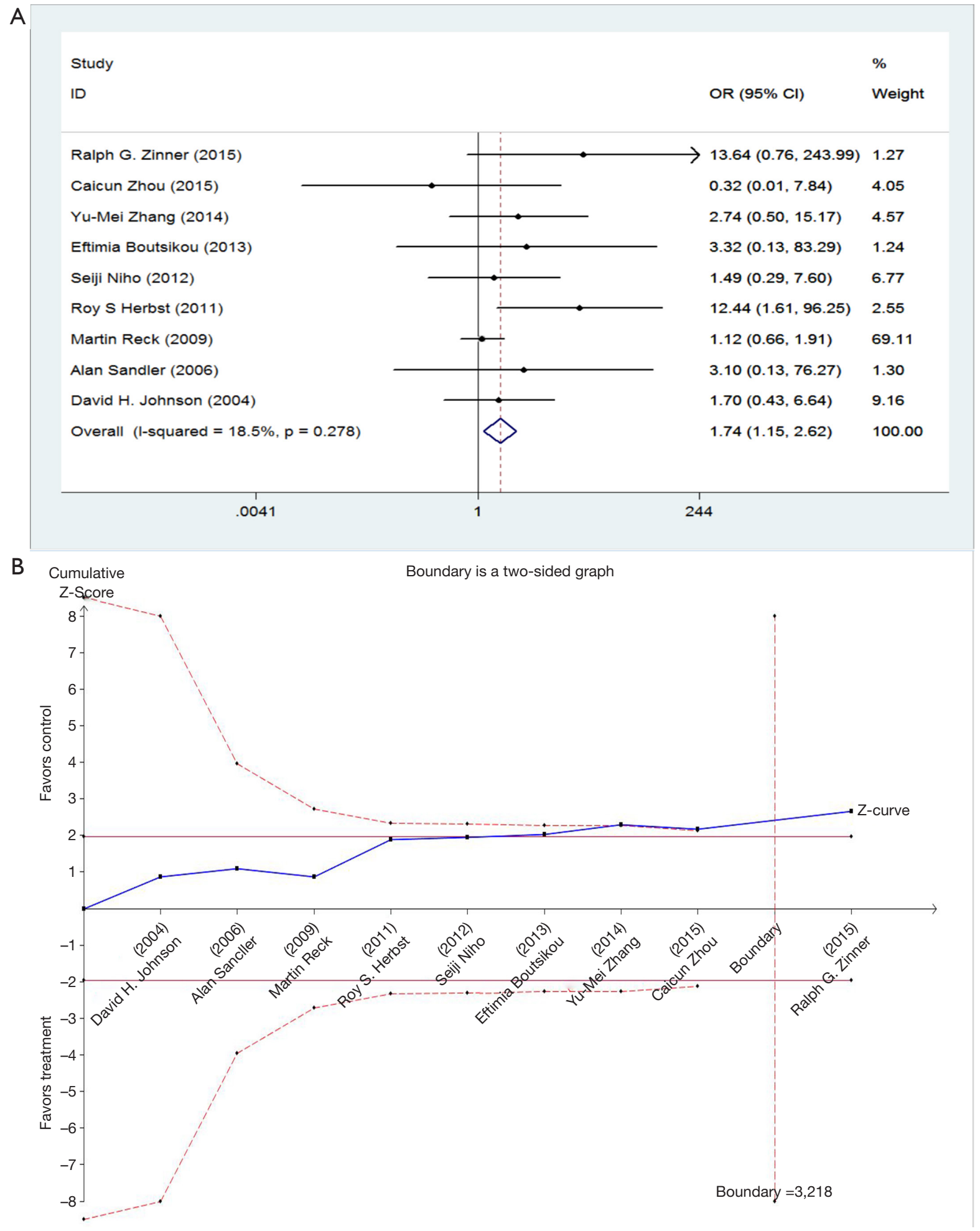

Figure 3 The forest pot and TSA of the odds ratio of bevacizumab-associated thromboembolism events. (A) The forest pot for metaanalysis of the ORs of bevacizumab-associated thromboembolism events; (B) Z-curve crossed the trial sequential monitoring boundary for hazard (red dotted curve) and the required sample size based on TSA ( $\mathrm{n}=3,687)$, entering the area of hazard (above the upper red line). OR, odds ratio; CI, confidence interval; TSA, trial sequence analysis. 


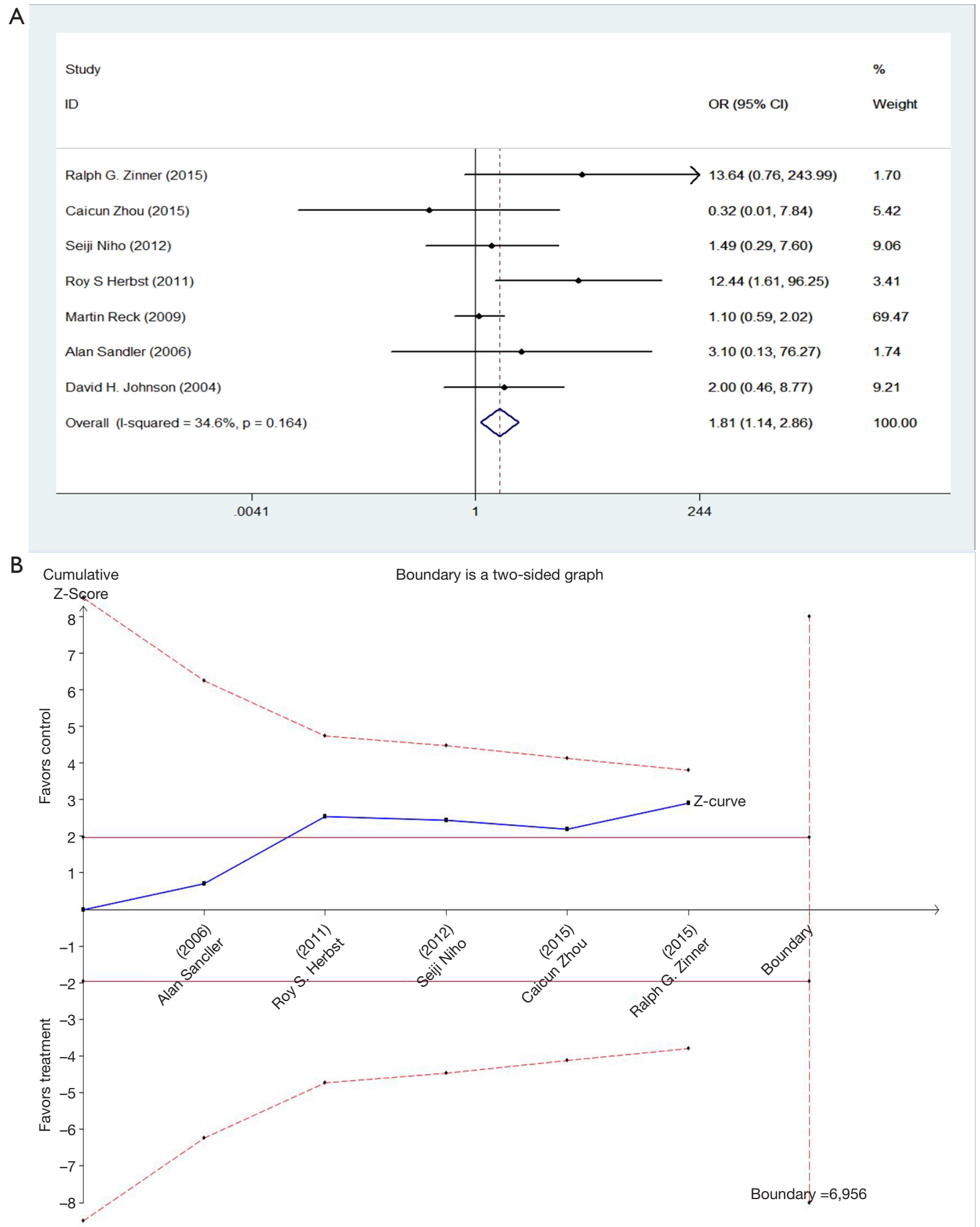

Figure 4 The forest pot and TSA of the odds ratio of high dose bevacizumab associated thromboembolism events. (A) OR of high dose bevacizumab associated thromboembolism events; (B) Z-curve did not cross the trial sequential monitoring boundary for hazard (red dotted curve) and did not reach the required sample size based on TSA ( $\mathrm{n}=3,007)$, entering the area of hazard (above the upper red line). OR, odds ratio; CI, confidence interval; TSA, trial sequence analysis. 
A

Study

ID

OR $(95 \% \mathrm{Cl}) \quad$ Weight

Eftimia Boutsikou (2013)

Yu-Mei Zhang (2014)

Martin Reck (2009)

David H. Johnson (2004)

Overall (I-squared $=0.0 \%, p=0.744)$

-

012

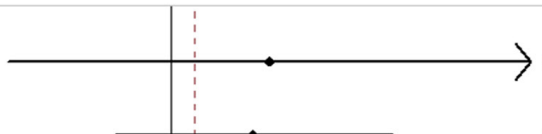

$3.32(0.13,83.29)$

1.91

$2.74(0.50,15.17) \quad 7.07$

$1.14(0.62,2.10) \quad 80.25$

$1.38(0.28,6.73) \quad 10.77$

$1.32(0.78,2.24) \quad 100.00$

\section{B Cumulative}

Z-Score

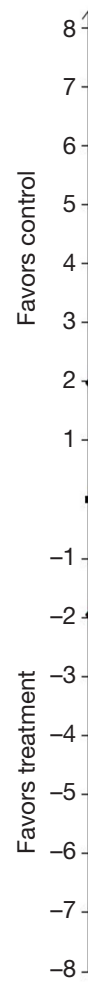

Boundary is a two-sided graph
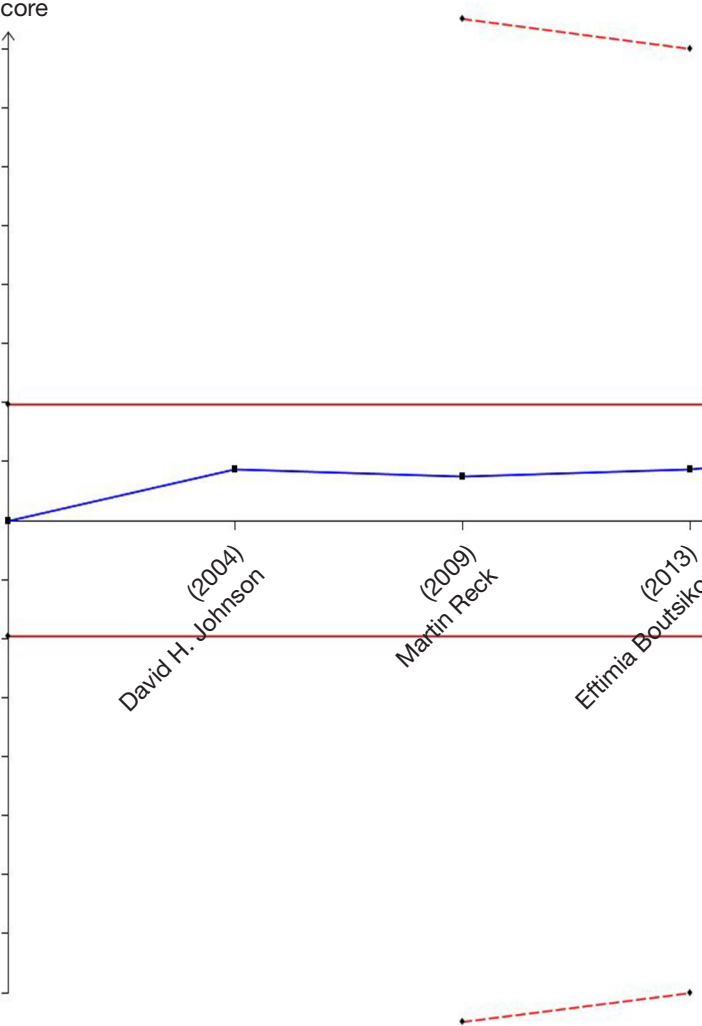

Boundary $=7,045$

Figure 5 The forest pot and TSA of the odds ratio of low dose bevacizumab associated thromboembolism events. (A) OR of low dose bevacizumab associated thromboembolism's events; (B) Z-curve did not cross the trial sequential monitoring boundary for hazard (red dotted curve) and did not reach the required sample size based on TSA ( $\mathrm{n}=846$ ), either entering the area of hazard (underneath the upper red line). OR, odds ratio; CI, confidence interval; TSA, trial sequence analysis. 


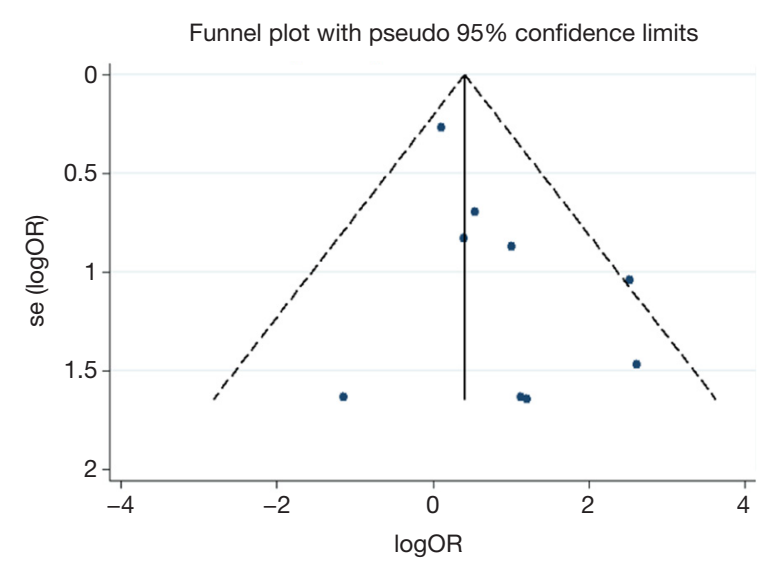

Figure 6 Funnel-plot standard error based on the OR for relative risk of thromboembolism. OR, odds ratio.

changed the ORs qualitatively, suggesting that our results were robust.

\section{Publication bias}

No evidence of publication bias was found for the OR of thromboembolism in our meta-analysis according to the funnel plot (Figure 6), and the Egger's test (95\% CI: -0.21 to $2.22, \mathrm{P}=0.092)$.

\section{Discussion}

Bevacizumab, an antibody that confers direct antiangiogenic effect, has been commonly prescribed as an anti-cancer agent in clinical practice. As a hallmark of cancer, angiogenesis is essential for tumor development. Anti-angiogenesis therapy, which acts on the tumor microenvironment, has been an efficient strategy in tumor treatment (7). Currently, bevacizumab has been approved for use in patients with NSCLC, colorectal cancer, breast cancer, brain glioblastoma and renal cancer by the US Food and Drug Administration (25). When focusing on NSCLC, we found that bevacizumab displayed a substantial benefit for these patients when combined with platinumbased chemotherapy regimen. In two phase III trials among patients in developed countries, the ECOG 4599 (8) and AVAiL trial (19), the addition of bevacizumab to standard first-line chemotherapy significantly improved PFS in patients with advanced NSCLC, but only ECOG 4599 showed a statistically significant benefit on OS compared with chemotherapy alone. Consistent treatment benefit among patients in Asian countries has also been shown in other two randomized clinical trials, BEYOND (24) and JO19907 (21). The combination of bevacizumab and carboplatin-paclitaxel resulted in a clinically significant prolonged PFS in both studies, and there was also a notable OS benefit in Chinese patients from the BEYOND trial. All these trials have formed the basis of general application of bevacizumab for the treatment of advanced NSCLC in many countries.

The recommended doses of bevacizumab approved by national authorities are 7.5 and $15 \mathrm{mg} / \mathrm{kg}$ given once every 3 weeks. Though the effectiveness of bevacizumab in advanced NSCLC has been validated, the dose-effect relationship has not been proven in vivo and in clinical practice (25). In the AVAiL trial (19), the hazard ratio for PFS was 0.75 (95\% CI: 0.62-0.91, P=0.003) for the lowdose $(7.5 \mathrm{mg} / \mathrm{kg}$ ) bevacizumab group versus placebo (median PFS, 6.7 vs. 6.1 months) and 0.82 (95\% CI: 0.68-0.98, $\mathrm{P}=0.03)$ for the high-dose $(15 \mathrm{mg} / \mathrm{kg})$ bevacizumab group versus placebo (median PFS, 6.5 vs. 6.1 months). In the AVF-0757g trial (11), only patients treated with highdose bevacizumab regimen had a significant longer median time to progression $(\mathrm{P}=0.023)$ versus control group (7.4 vs. 4.2 months, respectively). Both two studies lacked sufficient power to make a definitive conclusion regarding the relationship between the dose and efficacy directly. While in the previous meta-analysis (26), including the AVAiL and AVF-0757g trials, bevacizumab significantly prolonged the OS and PFS when added to platinum-based chemotherapy in patients with NSCLC and no significant difference between the high and low doses was observed.

For better application of bevacizumab, physicians should determine the safety of different treatment regimens. The most common bevacizumab-related AEs are often considered as grade 3 or severer forms (i.e., proteinuria, hypertension, haemorrhagic events, neutropenia) (13-15,27). Consistent with our results, several meta-analyses found that bevacizumab therapy was also a risk factor of thromboembolism (13,27-29). Thromboembolism, including venous thromboembolism (VTE) and arterial thromboembolism (ATE), is not a negligible AEs of bevacizumab despite of its low incidence. Because ATE can be life-threatening and may cause drug-related deaths $(18,20)$, such as the grade 5 AEs, so more attention should be paid. Nalluri et al. (28) concluded that patients with cancer treated with bevacizumab had a significantly increased risk for both all-grade and high-grade VTE ( $R R=1.33,95 \%$ CI: $1.13-1.56 ; \mathrm{P}<0.001)$. On the contrary, in Scappaticci 
et al.'s (29) analysis, combination with bevacizumab was associated with an increased risk of ATE (HR $=2.0,95 \% \mathrm{CI}$ : 1.05-3.75; P=0.031) but not VTE. However, both analyses included patients with various types of solid tumors. Gadgeel et al. (27) collated the safety data with bevacizumab in NSCLC revealing that patients were at risk of VTE but without taking the dose effect into account.

To our knowledge, this is the first study to estimate the incidence and risk of thromboembolism associated with bevacizumab therapy in patients with NSCLC by taking dosage into consideration. Comparing bevacizumab plus chemotherapy or erlotinib treatment regimen (experiment group) with chemotherapy or erlotinib alone (control group), the incidence of thromboembolism, was $4.8 \%$ (95\% CI: $1.9-7.7 \%$ ), was $2.9 \%$ (95\% CI: $0.6-5.1 \%$ ), respectively. We found that treatment with bevacizumab has a significantly higher risk of thromboembolic events $(\mathrm{OR}=1.74 ; 95 \% \mathrm{CI}: 1.15-2.62 ; \mathrm{P}=0.008)$ in patients with NSCLC. After analyses, we unexpectedly unraveled the different bevacizumab-related risk of thrombosis events between two doses. In the low-dose group $(7.5 \mathrm{mg} / \mathrm{kg}$ for three weeks), meta-analysis showed no significant difference between cases and controls ( $\mathrm{OR}=1.32$; 95\% CI: 0.78-2.24; $\mathrm{P}=0.296)$. However, in the high-dose group (15 mg/kg for three weeks), bevacizumab significantly increased the risk of thromboembolism ( $\mathrm{OR}=1.81 ; 95 \%$ CI: $1.14-2.86 ; \mathrm{P}=0.012)$. The result suggests that there may be a dose-toxicity relationship. A same conclusion according to the TSA and meta-analysis findings: the risk of thromboembolism was statistically significant and had a dose-dependent relationship. The pooled result was robust for the main study populations, However, subgroup result indicated that we still need further studies to confirm the dose-dependent effect of bevacizumab on the risk of thromboembolism.

In clinical practice, only European authorities have approved the low dose for lung cancer although no phase III study or meta-analysis has proved the superiority for high dose administration (25). Our findings confirm that bevacizumab at low dose may not be harmful to NSCLC patients when assessing the thrombosis risk. There is a robust evidence suggesting that low-dose bevacizumab should be recommended, concerning the use of bevacizumab in colorectal, lung and first-line ovarian cancer (25), which is consistent with findings from our study.

VTE, the most common thromboembolic event defined as deep vein thrombosis, pulmonary embolism, and central venous catheter-associated thrombosis, is an important and growing public health problem globally. In the US, VTE has an annual incidence of 1-2 per 1,000 of the general population, corresponding to 300,000-600,000 cases each year (30). However, the incidence rate of VTE is higher in patients with cancer than in general population and it is the second leading cause of death in patients with cancer (31). The risk of VTE depends on the types of cancer, with lung cancer being at greatest risks $(32,33)$. Furthermore, the absolute incidence of VTE in lung cancer was higher than the others due to the higher lung cancer prevalence (32). In lung cancer patients, risk factors of VTE consist of various treatment-, cancer-, and patient-related factors. The risk of VTE among cancer patients was four to seven times higher than that general population (34). Malignancy is associated with thromboembolic events because of increasing blood coagulability in patients with cancer (34). Differences of the primary site of cancer, tumor stage, and tumor histology contribute to different risks of developing VTE. NSCLC patients with adenocarcinoma and advanced cancer may more frequently develop VTE (32). When administrating medical treatment, both specific anti neoplastic agents and supportive care agents (particular bevacizumab) may have increased the risk $(32,35)$. Lung cancer patients who suffer from thromboembolism events have higher mortality rates, more economic costs and greater disability upon discharge than those without such complications (33). Thromboembolism in lung cancer patients is associated with substantial adverse outcomes and poor prognosis, therefore proper prophylaxis and management are urgently needed. On one hand, some biomarkers and predictors of thromboembolism have been investigated, with D-dimer $(32,36)$ and EGFR mutation being regarded as the most promising and reproducible ones, which may help to early identify thromboembolism events in clinical practice. Furthermore, low-molecular-weight heparins (LMWH) and direct oral anticoagulants have been used for treatment of the established thromboembolism $(35,37)$. Effective prophylaxis and proper treatment of VTE play an important role in cancer management for the benefits of reducing morbidity and mortality, and improving quality of life (38). In patients with lung cancer, LMWH is effective and safety for VTE prophylaxis and treatment. Farge et al. (37) compared anticoagulant prophylaxis with placebo or no intervention in ambulatory patients receiving systemic anticancer therapy. They found that $\mathrm{LMWH}$ significantly reduced the VTE risk by $50.3-53.6 \%$ in patients with lung cancer (1,926-2,075 cases). Moreover, thrombosis in cancer tends to recur and there have been 
data indicating that $\mathrm{LMWH}$ reduces the rate of recurrence of VTE (38). In addition, the rate of major bleeding increased in patients treated with anticoagulants, though there were survival benefits (39). Hence, appropriate risk stratification based on patients' characters and treatment strategy is of great importance on the improvement of risk-benefit and cost-effectiveness. There are several limitations of our study. First, there were only four trials which included 846 patients studying the effects of lowdose bevacizumab. Therefore, we could not detect the risk of thromboembolism with sufficient statistical power, which may have increased the bias. Second, we did not perform subgroup analysis different levels of thromboembolism events, because few trials showed clear grading, we could not obtain sufficient data for subgroup analysis. Third, the incidence of thromboembolism events was low, and we have also excluded trials which did not show events of thromboembolism, although no publication bias was identifies for the OR, the effect sizes of thromboembolism risk of bevacizumab might still have been overestimated. Finally, high-quality studies published in other languages might not have been included, therefore our search may be biased.

\section{Conclusions}

Our study suggested that bevacizumab treatment in NSCLC patients may predispose to thromboembolism with some dose-relevant toxicity effects, and that high dosage may confer higher risk of thromboembolism. Therefore, low-dose bevacizumab may be preferable for patients with NSCLC, who have lower risks of developing thromboembolism

\section{Acknowledgements}

Funding: This study was supported by Science and Technology Planning Project of Guangzhou City, China (No. 201607010374); Science and Technology Planning Project of Guangdong Province, China (No. 2014A020212562); National Natural Science Foundation of China (No. 81670036).

\section{Footnote}

Conflicts of Interest: The authors have no conflicts of interest to declare.

\section{References}

1. Siegel RL, Miller KD, Jemal A. Cancer Statistics, 2017.
CA Cancer J Clin 2017;67:7-30.

2. Planchard D. Bevacizumab in non-small-cell lung cancer: a review. Expert Rev Anticancer Ther 2011;11:1163-79.

3. Botrel TE, Clark O, Clark L, et al. Efficacy of bevacizumab (Bev) plus chemotherapy (CT) compared to CT alone in previously untreated locally advanced or metastatic nonsmall cell lung cancer (NSCLC): systematic review and meta-analysis. Lung Cancer 2011;74:89-97.

4. Gentzler RD, Yentz SE, Patel JD. Bevacizumab in advanced NSCLC: chemotherapy partners and duration of use. Curr Treat Options Oncol 2013;14:595-609.

5. Greillier L, Tomasini P, Barlesi F. Bevacizumab in the treatment of nonsquamous non-small cell lung cancer: clinical trial evidence and experience. Ther Adv Respir Dis 2016;10:485-91.

6. Manegold C. Bevacizumab for the treatment of advanced non-small-cell lung cancer. Expert Rev Anticancer Ther 2008;8:689-99.

7. Cabebe $\mathrm{E}, \mathrm{Wakelee} \mathrm{H}$. Role of anti-angiogenesis agents in treating NSCLC: focus on bevacizumab and VEGFR tyrosine kinase inhibitors. Curr Treat Options Oncol 2007;8:15-27.

8. Sandler A, Gray R, Perry MC, et al. Paclitaxel-carboplatin alone or with bevacizumab for non-small-cell lung cancer. N Engl J Med 2006;355:2542-50.

9. Wagstaff AJ, Keam SJ, McCormack PL. Bevacizumab plus platinum-based chemotherapy: in advanced non-small cell lung cancer. BioDrugs 2009;23:187-96.

10. Gautschi O, Rothschild SI, Li Q, et al. Bevacizumab Plus Pemetrexed Versus Pemetrexed Alone as Maintenance Therapy for Patients With Advanced Nonsquamous NonSmall-cell Lung Cancer: Update From the Swiss Group for Clinical Cancer Research (SAKK) 19/09 Trial. Clin Lung Cancer 2017;18:303-9.

11. Johnson DH, Fehrenbacher L, Novotny WF, et al. Randomized phase II trial comparing bevacizumab plus carboplatin and paclitaxel with carboplatin and paclitaxel alone in previously untreated locally advanced or metastatic non-small-cell lung cancer. J Clin Oncol 2004;22:2184-91.

12. Mok TS, Hsia TC, Tsai CM, et al. Efficacy of bevacizumab with cisplatin and gemcitabine in Asian patients with advanced or recurrent non-squamous non-small cell lung cancer who have not received prior chemotherapy: a substudy of the Avastin in Lung trial. Asia Pac J Clin Oncol 2011;7 Suppl 2:4-12.

13. Chen Z, Zhong B, Lun X, et al. Specific Safety Profile of Bevacizumab in Asian Patients With Advanced NSCLC: A 
Meta-Analysis. Medicine (Baltimore) 2015;94:e975.

14. Cao C, Wang J, Bunjhoo H, et al. Risk profile of bevacizumab in patients with non-small cell lung cancer: a meta-analysis of randomized controlled trials. Acta Oncol 2012;51:151-6.

15. Lai XX, Xu RA, Yu-Ping L, et al. Risk of adverse events with bevacizumab addition to therapy in advanced nonsmall-cell lung cancer: a meta-analysis of randomized controlled trials. Onco Targets Ther 2016;9:2421-8.

16. Qi WX, Min DL, Shen Z, et al. Risk of venous thromboembolic events associated with VEGFR-TKIs: a systematic review and meta-analysis. Int J Cancer 2013;132:2967-74.

17. Higgins JPT, Green S. editors. Cochrane Handbook for Systematic Reviews of Interventions Version 5.1.0 [updated March 2011]. The Cochrane Collaboration, 2011. Available online: http://handbook.cochrane.org.

18. Zinner RG, Obasaju CK, Spigel DR, et al. PRONOUNCE: randomized, open-label, phase III study of first-line pemetrexed + carboplatin followed by maintenance pemetrexed versus paclitaxel + carboplatin + bevacizumab followed by maintenance bevacizumab in patients ith advanced nonsquamous non-small-cell lung cancer. J Thorac Oncol 2015;10:134-42.

19. Reck M, von Pawel J, Zatloukal P, et al. Phase III trial of cisplatin plus gemcitabine with either placebo or bevacizumab as first-line therapy for nonsquamous non-small-cell lung cancer: AVAil. J Clin Oncol 2009;27:1227-34.

20. Herbst RS, Ansari R, Bustin F, et al. Efficacy of bevacizumab plus erlotinib versus erlotinib alone in advanced non-small-cell lung cancer after failure of standard first-line chemotherapy (BeTa): a double-blind, placebocontrolled, phase 3 trial. Lancet 2011;377:1846-54.

21. Niho S, Kunitoh H, Nokihara H, et al. Randomized phase II study of first-line carboplatin-paclitaxel with or without bevacizumab in Japanese patients with advanced non-squamous non-small-cell lung cancer. Lung Cancer 2012;76:362-7.

22. Boutsikou E, Kontakiotis T, Zarogoulidis P, et al. Docetaxel-carboplatin in combination with erlotinib and/or bevacizumab in patients with non-small cell lung cancer. Onco Targets Ther 2013;6:125-34.

23. Zhang YM, Li YQ, Liu ZH, et al. Clinical efficacy of bevacizumab concomitant with pemetrexed in patients with advanced non-small cell lung cancer. Asian Pac J Cancer Prev 2014;15:3447-50.

24. Zhou C, Wu YL, Chen G, et al. BEYOND: A
Randomized, Double-Blind, Placebo-Controlled, Multicenter, Phase III Study of First-Line Carboplatin/ Paclitaxel Plus Bevacizumab or Placebo in Chinese Patients With Advanced or Recurrent Nonsquamous NonSmall-Cell Lung Cancer. J Clin Oncol 2015;33:2197-204.

25. Falk AT, Barriere J, Francois E, et al. Bevacizumab: A dose review. Crit Rev Oncol Hematol 2015;94:311-22.

26. Soria JC, Mauguen A, Reck M, et al. Systematic review and meta-analysis of randomised, phase II/III trials adding bevacizumab to platinum-based chemotherapy as first-line treatment in patients with advanced non-small-cell lung cancer. Ann Oncol 2013;24:20-30.

27. Gadgeel SM. Safety profile and tolerability of antiangiogenic agents in non-small-cell lung cancer. Clin Lung Cancer 2012;13:96-106.

28. Nalluri SR, Chu D, Keresztes R, et al. Risk of venous thromboembolism with the angiogenesis inhibitor bevacizumab in cancer patients: a meta-analysis. JAMA 2008;300:2277-85.

29. Scappaticci FA, Skillings JR, Holden SN, et al. Arterial thromboembolic events in patients with metastatic carcinoma treated with chemotherapy and bevacizumab. J Natl Cancer Inst 2007;99:1232-9.

30. Chaffey P, Thompson M, Pai AD, et al. Usefulness of Statins for Prevention of Venous Thromboembolism. Am J Cardiol 2018;121:1436-40.

31. Levitan N, Dowlati A, Remick SC, et al. Rates of initial and recurrent thromboembolic disease among patients with malignancy versus those without malignancy. Risk analysis using Medicare claims data. Medicine (Baltimore) 1999;78:285-91.

32. Ay C, Unal UK. Epidemiology and risk factors for venous thromboembolism in lung cancer. Curr Opin Oncol 2016;28:145-9.

33. Steuer CE, Behera M, Kim S, et al. Predictors and outcomes of venous thromboembolism in hospitalized lung cancer patients: A Nationwide Inpatient Sample database analysis. Lung Cancer 2015;88:80-4.

34. Ohashi Y, Ikeda M, Kunitoh H, et al. Venous thromboembolism in patients with cancer: design and rationale of a multicentre, prospective registry (CancerVTE Registry). BMJ Open 2018;8:e018910.

35. Salla E, Dimakakos EP, Tsagkouli S, et al. Venous Thromboembolism in Patients Diagnosed With Lung Cancer. Angiology 2016;67:709-24.

36. Alexander M, Burbury K. A systematic review of biomarkers for the prediction of thromboembolism in lung cancer - Results, practical issues and proposed 
strategies for future risk prediction models. Thromb Res 2016;148:63-9.

37. Farge D, Bounameaux H, Brenner B, et al. International clinical practice guidelines including guidance for direct oral anticoagulants in the treatment and prophylaxis of venous thromboembolism in patients with cancer. Lancet Oncol 2016;17:e452-66.

Cite this article as: Li LJ, Chen DF, Wu GF, Guan WJ, Zhu Z, Liu YQ, Gao GY, Qin YY, Zhong NS. Incidence and risk of thromboembolism associated with bevacizumab in patients with non-small cell lung carcinoma. J Thorac Dis 2018;10(8):50105022. doi: $10.21037 /$ jtd.2018.07.09
38. Ikushima S, Ono R, Fukuda K, et al. Trousseau's syndrome: cancer-associated thrombosis. Jpn J Clin Oncol 2016;46:204-8.

39. Zhang J, Zhang YL, Ma KX, et al. Efficacy and safety of adjunctive anticoagulation in patients with lung cancer without indication for anticoagulants: a systematic review and meta-analysis. Thorax 2013;68:442-50. 